\title{
Post-treatment parenthood in Hodgkin's lymphoma survivors
}

\author{
CE Kiserud ${ }^{*, 1}$, A Fosså ${ }^{2}$, H Holte ${ }^{2}$ and SD Fosså ${ }^{1,3}$ \\ 'Unit for long term outcome, Department of Clinical Cancer Research, Rikshospitalet-Radiumhospitalet Medical Center, Oslo, Norway; ${ }^{2}$ Cancer Clinic, \\ Rikshospitalet-Radiumhospitalet Medical Center, 0310 Oslo, Norway; ${ }^{3}$ University of Oslo, Oslo, Nonway
}

Attempted and achieved post-treatment parenthood, with or without use of assisted reproduction techniques (ARTs), was assessed in Hodgkin's lymphoma survivors treated from 197I-1998, aged below 50 (females) or 65 (males) at diagnosis, aged 18 to 75 at survey. Four treatment groups were constructed: radiotherapy only, low -, medium - and high gonadotoxic chemotherapy (with or without radiotherapy in the three chemotherapy groups). Using Kaplan-Meier estimates, log-rank tests and Cox regression analyses, factors influencing post-treatment parenthood were investigated, with birth of the first child after treatment as the end point. Fortyfive per cent (120/269) of males and 50\% (9I//84) of females reported attempted post-treatment parenthood. Of these, 76 (63\%) males and 68 (75\%) females had a child without use of ARTs. In addition 10 males and one female achieved post-treatment parenthood with use of ARTs. Treatment group was significantly associated with post-treatment parenthood, with highest probabilities after radiotherapy only and low gonadotoxic chemotherapy. In univariate analyses, age at diagnosis was a significant factor related to post-treatment parenthood in females.

British Journal of Cancer (2007) 96, |442- |449. doi: I0.1038/sj.bjc.66037I I www.bjcancer.com

Published online 3 April 2007

(c) 2007 Cancer Research UK

Keywords: Hodgkin's lymphoma; chemotherapy; radiotherapy; post-treatment parenthood

Most previous studies on fertility in Hodgkin's lymphoma survivors (HLSs) have addressed post-treatment spermatogenesis and secondary amenorrhea (Viviani et al, 1985, 1991; Hill et al, 1995; Behringer et al, 2005). Although the number of achieved pregnancies and childbirths is sometimes reported in small series (Schilsky et al, 1981; Whitehead et al, 1983; Bonadonna et al, 1984; Anselmo et al, 1990), the selection of survivors attempting parenthood is not reported, with a few exceptions (Aisner et al, 1993; Hodgson et al, 2006).

We therefore aimed to determine rates for attempted and achieved post-treatment parenthood with or without use of assisted reproduction techniques (ARTs), in HLSs treated at the Rikshospitalet-Radiumhospitalet Medical Center (RRMC).

\section{PATIENTS AND METHODS}

\section{Patients}

During 1971 - 1998, a total of 1567 HL patients were registered in the lymphoma database of the RRMC. A questionnaire survey concerning various late effects and quality of life was performed from 2001 to 2002 among consecutive HLSs fulfilling the following criteria: treatment from 1971 to 1998 , age $18-75$ years at the time of survey, no relapse after 1 January 1999, no secondary cancer

*Correspondence: Dr CE Kiserud, Unit for long term outcome, Department of Clinical Cancer Research, Rikshospitalet-Radiumhospitalet Medical Center, 0310 Oslo, Norway.

E-mail: cecilie.essholt.kiserud@radiumhospitalet.no

Received 2 January 2007; revised 23 February 2007; accepted I March 2007; published online 3 April 2007 (except cutaneous basal cell carcinoma) and valid postal address. The present study addresses the survivors' achievement of posttreatment parenthood, as evidenced in the survey, and excluded females aged above 50 years and males above 65 years at diagnosis. Patients diagnosed with a secondary non-Hodgkin's lymphoma (NHL) were included as their treatment options were similar to those with relapsed HL. For supplementary information on fertility, the female responders from 2002 were contacted again in 2005. The present study includes male responders from the survey of 2002 and female responders from the survey of 2005 (with their reproduction as of 2002) (Figure 1).

\section{Treatment principles}

The treatment strategies of HL at the RRMC have been described previously (Abrahamsen et al, 1996, 1997; Holte et al, 1996; Aurlien et al, 1998; Blystad et al, 2001a, 2001b) and are summarized in Table 1a and b. From 1985 to 1990, primary chemotherapy with MVPP/ChlVPP was gradually replaced by ABOD or EBVP for limited disease. At relapse, patients were treated with noncross-resistant chemotherapy, or - from 1990 with high-dose chemotherapy with autologous stem cell support (HDT). Fractionated total body irradiation (TBI) with high-dose cyclophosphamide was used as a conditioning regimen for HDT until 1995, and was thereafter changed to chemotherapy only (BEAC/BEAM) (Blystad et al, 2001a, 2001b).

In stage I and II patients, mantle field and inverted Y field radiotherapy dominated up until 1997 with target doses of $41.4 \mathrm{~Gy}$ $(1.8 \mathrm{~Gy} \times 23)$ given alone or after chemotherapy. If possible, the standard inverted Y field was modified (to unilateral L-field or para-aortic field) in order to reduce the gonadal doses. Patients with stage III and IV received radiotherapy to areas of initial bulky 
tumours or residual masses after chemotherapy. Gonadal shielding was routinely used, reducing gonadal doses from 0.6 to $0.1-0.2 \mathrm{~Gy}$ during mantle field irradiation. Inverted $\mathrm{Y}$ field resulted in
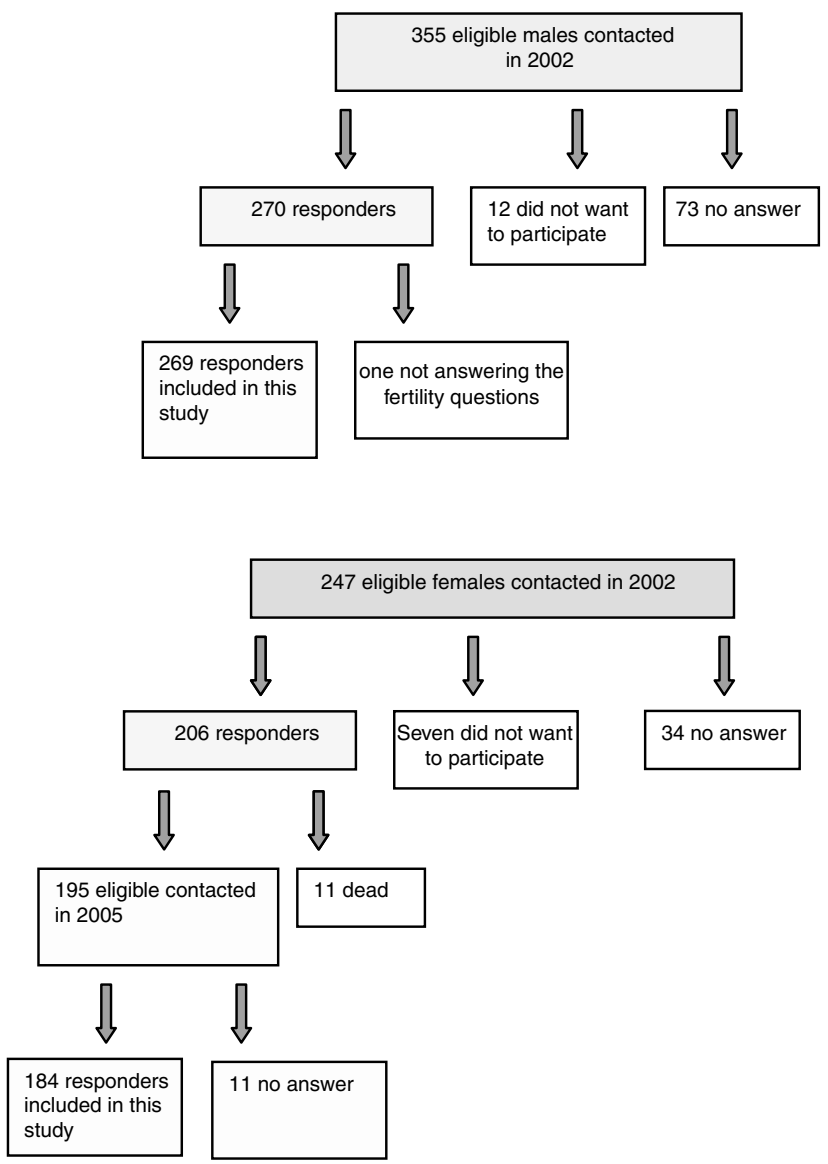

Figure I Flow charts of male and female HLSs who met the inclusion criteria for the present study. testicular doses of $0.6-0.9 \mathrm{~Gy}$. In the $1970 \mathrm{~s}$, medial transposition of the ovaries was offered to young patients. In these patients, the shielded ovaries received about 3 Gy when treated with inverted Y field (Jetne, RRMC, 1972, unpublished data).

For the purpose of this study, the total treatment for each patient as registered in the lymphoma database was summarised. Chemotherapy was sub-grouped according to the expected gonadotoxicity of the regimens used: low (LowChem), medium (MedChem) and high (HighChem) gonadotoxicity (Viviani et al, 1985; Howell and Shalet, 2001; Behringer et al, 2005; American Society of Clinical Oncology et al, 2006; Hodgson et al, 2006).Two sub-groups of radiotherapy were defined: supradiaphragmatic radiotherapy (SupRad) only and infradiaphragmatic radiotherapy with or without supradiaphragmatic irradiation (InfRad). In preliminary analyses, no differences were observed between these two subgroups of radiotherapy as to attempted or achieved posttreatment parenthood. Therefore, one treatment variable (treatment) was constructed discriminating patients with radiotherapy only from those having chemotherapy with low, medium and high gonadotoxicity (with or without radiotherapy).

\section{Assisted reproduction techniques}

The Norwegian legislation on ARTs has been rather restrictive. Since 1980, Norwegian male patients have had the option for pretreatment semen cryopreservation, with ARTs performed as intrauterine insemination (IUI) in the early 1980 s and as in vitro fertilisation (IVF) since 1988. Intracytoplasmatic sperm injection (ICSI) has been offered since 1995. Pretreatment cryopreservation of fertilised oocytes has been offered since 1988, but has never been used by any cancer patient. Use of donated semen, but not oocytes, is allowed.

\section{Questionnaire, data management and ethical considerations}

The questionnaire included instruments on fatigue and quality of life as reported previously (Hjermstad et al, 2005, 2006), in addition to questions concerning reproduction relevant for the present analysis. We assessed attempted and achieved

Table I Treatment of Hodgkin's lymphoma at the RRMC 1970-1998

\begin{tabular}{|c|c|c|c|}
\hline & Chemotherapy & Radiotherapy (RT) & Dose and fractions of radiotherapy \\
\hline \multicolumn{4}{|c|}{$\begin{array}{l}\text { (a) Treatment principles } \\
\text { Stage I \& II }\end{array}$} \\
\hline $1970-1980$ & & RT only Extended fields MF/lnv Y & $2 \mathrm{~Gy} \times 20$ \\
\hline $1980-1988$ & $\begin{array}{l}\text { With risk factors: four MVPP/ChIVPP } \\
\text { before RT }\end{array}$ & Extended fields MF/Inv Y & $2 \mathrm{~Gy} \times 20$, from $19821.8 \mathrm{~Gy} \times 23$ \\
\hline $1988-1997$ & With risk factors: 2-4 EBVP before RT & Extended fields MF/lnv Y & $1.8 \mathrm{~Gy} \times 23$ \\
\hline \multicolumn{4}{|l|}{ Stage III and IV } \\
\hline $1970-1985$ & Eight MVPP/ChIVPP & $\begin{array}{l}\text { To bulky tumor or residual mass TNI to } \\
\text { some stage III patients until } 1980\end{array}$ & $2 \mathrm{~Gy} \times 20$ \\
\hline$|985-199|$ & $\begin{array}{l}\text { Eight ChIVPP or } 8 \text { ABOD/ChIVPP } \\
\text { (alternating) }\end{array}$ & To bulky tumor or residual mass & $2 \mathrm{~Gy} \times 20$ or $1.8 \mathrm{~Gy} \times 23$ \\
\hline $1992-1998$ & $8 \mathrm{ABVD}$ & To bulky tumor or residual mass & 2 Gy $\times 20$ or 1,8 Gy $\times 23$ \\
\hline Low & Medium & High & \\
\hline \multicolumn{4}{|c|}{ (b) Chemotherapy and expected gonadal toxicity } \\
\hline $\mathrm{ABO}(\mathrm{V}) \mathrm{D}$ & ChIVPP & ChIVPP & \\
\hline \multirow[t]{4}{*}{ EBVP } & MVPP & MVPP & \\
\hline & Medium if $\leqslant 4$ courses & High if $>4$ courses & \\
\hline & $\mathrm{CHOP}$ & $\begin{array}{l}\text { High-dose chemotherapy with autologous } \\
\text { stem cell support BEAC/BEAM }\end{array}$ & \\
\hline & MIME & & \\
\hline
\end{tabular}

MF = mantle field; RRMC = Rikshospitalet-Radiumhospitalet Medical Center; Inv $\mathrm{Y}=$ inverted $\mathrm{Y}$ field; TNI = total nodal irradiation. For details on chemotherapy: Aurlien et al (1998); Diehl (200I); Mills et al (1995). 
post-treatment parenthood, number of pre- and post-treatment children, calendar years of childbirths and use of ARTs (without differentiation between IUI, IVF and ICSI and without recording the use of hormone therapy alone for inducing ovulation). Data from the questionnaire survey were merged with the lymphoma database of the RRMC.

Informed consent was obtained from all responders. The participants were informed in the invitation letter that the survey included various late effects after cancer treatment and quality of life. The Regional Committee for Medical Research Ethics, Health Region South, Norway and the Institutional Review Board at the RRMC approved the study.

\section{Statistics}

Data were analysed using descriptive statistics (SPSS 13.0 for PC).

Factors influencing attempted post-treatment parenthood were analysed by cross-tables and $\chi^{2}$ tests for categorical variables, and by $t$-test for continuous variables. Binary logistic regression analyses were carried out with attempted post-treatment parenthood regarded as the dependent factor.

In patients attempting post-treatment parenthood KaplanMeier estimates, log-rank tests and Cox regression analyses evaluated the probability of becoming parents without use of ARTs, with the first post-treatment childbirth as the end point. The observation time for post-treatment parenthood started 8 months after start of last treatment and ended at the date of first childbirth, or 30 June 2002 (the cutoff date of the study), whichever occurred first. For Kaplan-Meier analyses, patients were categorised into three groups according to age at diagnosis: (I): $\leqslant 20.0$ years, (II): 20.1-30.0 years, (III): $30.1-40.0$ years at diagnosis. In Cox regression analyses, age at diagnosis was used as a continuous variable because of lack of proportionality in the Kaplan-Meier curve. Variables significant in univariate analyses were included as covariates in multivariate analyses together with clinical parameters of major interest. $P$-values less than 0.05 (two-tailed) were considered significant, and $95 \%$ confidence intervals are given.

\section{RESULTS}

\section{Patients' characteristics}

Of the $1567 \mathrm{HL}$ patients registered in the database from this period, the 602 eligible for the present study were contacted by mail ( 811 were dead at the time of the survey, 107 had a second cancer diagnosis, 24 had relapse after 1999, 19 were excluded because of age criteria and four had no valid postal address). There were 269 male and 184 female responders (a response rate of $75 \%$ (Figure 1)). No differences in observation time, initial stage, principal therapeutic group and relapse rates were observed for responders and non-responders. Responding males were

Table 2 Patients characteristics

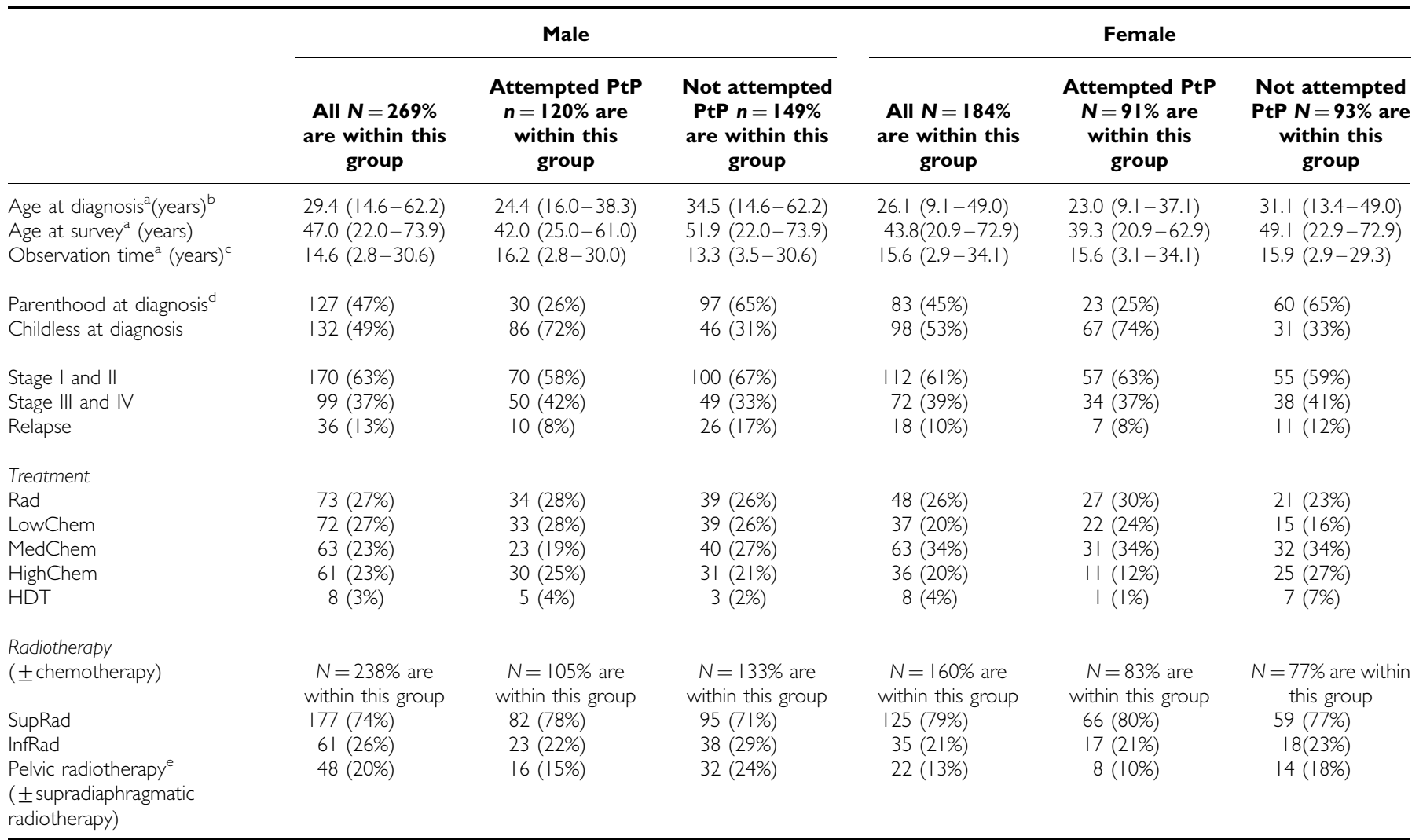

HDT = high-dose chemotherapy with autologous stem cell support; HighChem $=$ high gonadotoxic chemotherapy ( \pm radiotherapy); InfRad $=$ infradiaphragmatic radiotherapy \pm supradiaphragmatic radiotherapy; LowChem = low gonadotoxic chemotherapy ( \pm radiotherapy); MedChem $=$ medium gonadotoxic chemotherapy $( \pm$ radiotherapy); PtP $=$ post-treatment parenthood; Rad = radiotherapy only; SupRad, supradiaphragmatic radiotherapy only. ${ }^{\mathrm{a}}$ Median (range). ${ }^{\mathrm{b}}$ Significant factor with regard to attempted PtP. In both males and females, $P<0.00 \mathrm{I}$ in univariate analyses ( $t$-test), $P<0.00 \mathrm{I}$ in multivariate analyses. ${ }^{c}$ Observation time: time from diagnosis added 8 months, to time of the survey. ${ }^{d}$ Significant factor with regard to attempted PtP. In both males and females, $P<0.001$ in univariate analyses $\left(\chi^{2}\right.$ test), $P=0.027$ in multivariate analyses. ${ }^{e} P e l v i c$ radiotherapy: inverted $\mathrm{Y}$-, L-, Sacrum(skeletal)- fields, total body irradiation (TBI). 
significantly older than the non-responding males (29.4 vs 26.8 years (median)), whereas the opposite was the case in females (26.1 vs 32.3 years (median)).

Of all responders, $86 \%$ were $<40$ years at diagnosis. The median observation time from last treatment to survey was 15 years (range $3-34$ years), with $62 \%$ of the patients diagnosed before 1989 , and $61 \%$ treated with radiotherapy and chemotherapy. At the time of diagnosis, $46 \%$ had at least one child (127 males had 1-7 children and 83 females had 1-6 children) (Table 2).

\section{Attempted post-treatment parenthood}

Ninety-one (50\%) females and $120(45 \%)$ males reported to have attempted post-treatment parenthood, all aged below 40 years at diagnosis. In both univariate and multivariate analyses, low age and childlessness at diagnosis were the only variables associated with post-treatment attempts of parenting. Twentythree females reported not to have attempted post-treatment parenthood because they were convinced to be infertile after treatment; three of them were treated with radiotherapy only, 17 treated with medium or high and three with low gonadotoxic chemotherapy. The comparable number for males is not known.

\section{Post-treatment parenthood}

At the time of the survey, 68 females (75\%) and 76 males (63\%) who had attempted post-treatment parenthood had been successful without use of ARTs. The 10-year probability of post-treatment parenthood was $59 \%$ in females and $56 \%$ in males (no significant difference between genders) (Figure 2, Table 3). In patients childless at diagnosis, the 10 -year probabilities of post-treatment parenthood were $58 \%$ in females and $54 \%$ in males (data not shown).

Females aged below 30 years at diagnosis were significantly more likely to achieve post-treatment parenthood compared to older females. After 10 years, the group aged 20.1-30.0 at diagnosis had the highest probability of post-treatment parenthood: $77 \%$, compared to $50 \%$ in the youngest group and $18 \%$ in the oldest group. Fifteen years after diagnosis, the comparable figures were $89 \%$ in the group aged 20.1-30.0 at diagnosis and $85 \%$ in those aged $\leqslant 20$ years at diagnosis. Only two of 11 females older than 30 years at diagnosis achieved motherhood, one after mantle field irradiation only and one after four EBVP followed by mantle field irradiation. In males, there was no significant difference between the age groups.

Males, but not females, diagnosed after 1988 had significantly improved probability of achieving post-treatment parenthood compared to those diagnosed before 1989, with 10 -year probabilities of 81 vs $43 \%$. In males, but not in females, stage I/II was followed by significantly higher probability of post-treatment parenthood, compared to stage III/IV.

In males, there was a significantly higher probability of posttreatment fatherhood after radiotherapy only and after chemotherapy of low gonadotoxicity compared to chemotherapy with medium or high gonadotoxicity. The 10-year probabilities of achieving post-treatment parenthood in the various groups were 71 (Rad), 85 (LowChem), 35 (MedChem) and 18\% (HighChem).

In females, a significantly higher probability of achieving posttreatment motherhood was observed after radiotherapy only compared to chemotherapy with either medium or high gonadotoxicity. In addition, a comparable difference was seen between those treated with low and high gonadotoxic chemotherapy. The 10 -year probabilities were $82 \%$ (Rad), 55\% (LowChem), $51 \%$ (MedChem) and 27\% (HighChem).

Treatment with highly gonadotoxic chemotherapy did not exclude the possibility of having a child without the use of ARTs. Of 11 females attempting motherhood after highly gonadotoxic
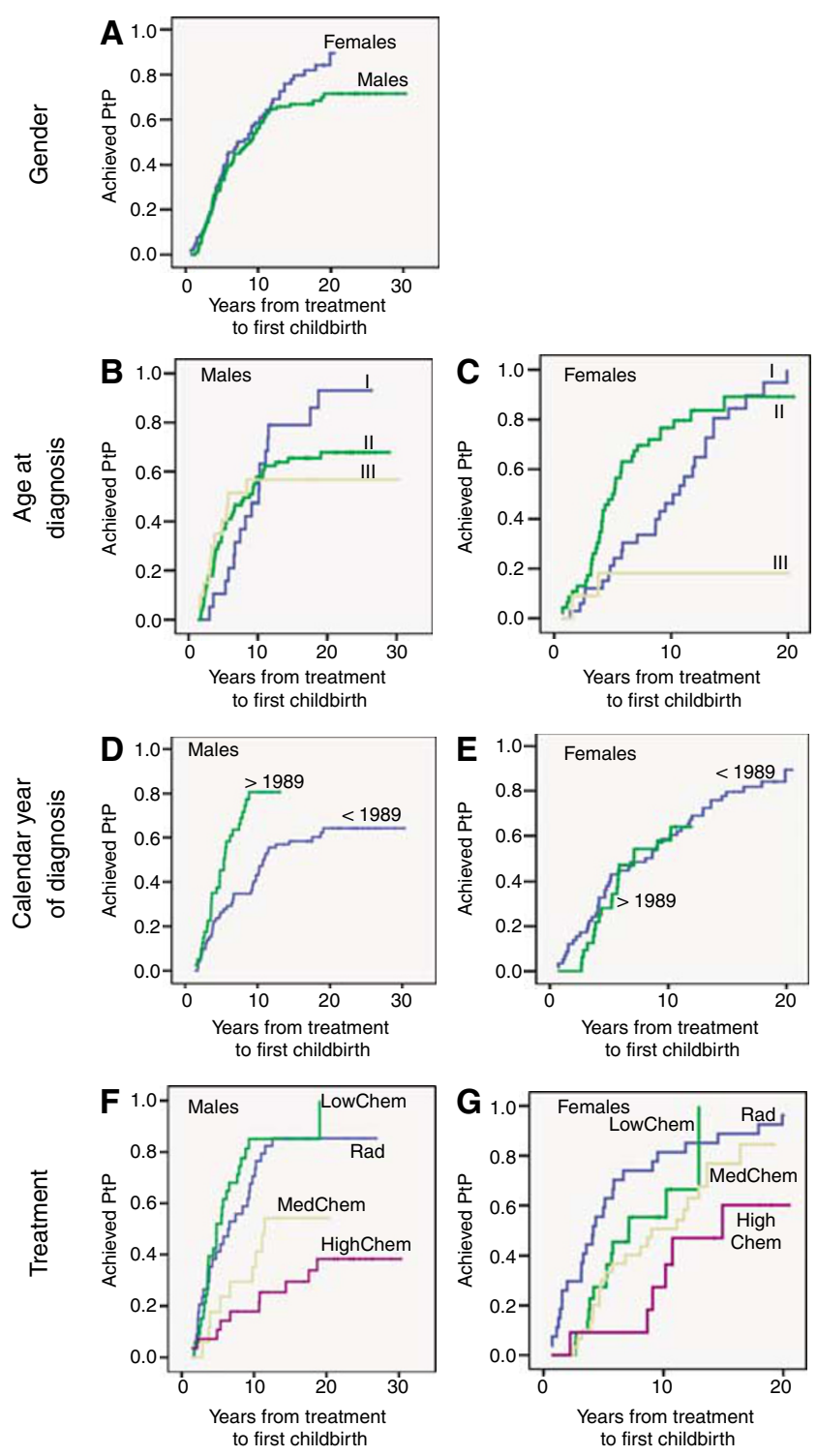

Figure 2 Achieved post-treatment parenthood (PtP) in male and female HLSs among those who attempted PtP, according to: (A) Gender. Males $n=112$, females $n=90$. (B and $\mathbf{C})$ Age at diagnosis. $(I) \leqslant 20$ years $(n=33$ females, $n=19$ males), (II) $20.1-30.0$ years ( $n=46$ females, $n=73$ males), (III) $30.1-40.0$ years ( $n=1 \mid$ females, $n=20$ males). (D and E) Calendar year of diagnosis. < 1989: diagnosis before 1989 ( $n=72$ males, $n=58$ females), > 1989: diagnosis in/after 1989 ( $n=40$ males, $n=32$ females). (F and $\mathbf{G})$ Treatment Rad = radiotherapy only $(n=34$ males, $n=27$ females), LowChem = low gonadotoxic chemotherapy $(n=33$ males, $n=22$ females), MedChem = medium gonadotoxic chemotherapy ( $n=17$ males, $n=30$ females), HighChem = high gonadotoxic chemotherapy ( $n=28$ males, $n=\mid$ I females).

chemotherapy, six were successful; all six were aged $<25$ years at diagnosis. The five patients who did not achieve post-treatment parenthood were 30-36 years at diagnosis (Table 4). Ten of 28 males attempting fatherhood after highly gonadotoxic chemotherapy became fathers. Two of these achieved children spontaneously 4 years after HDT with BEAM as conditioning regimen, one of them after having a first post-treatment child with the use of pretreatment cryopreserved semen.

Of all irradiated patients, $76 \%$ had received supradiaphragmatic radiotherapy only. Of 16 males and eight females who had 
Table 310 years probability of achieving PtP among those who attempted, excluding those achieving PtP with the use of assisted reproduction techniques

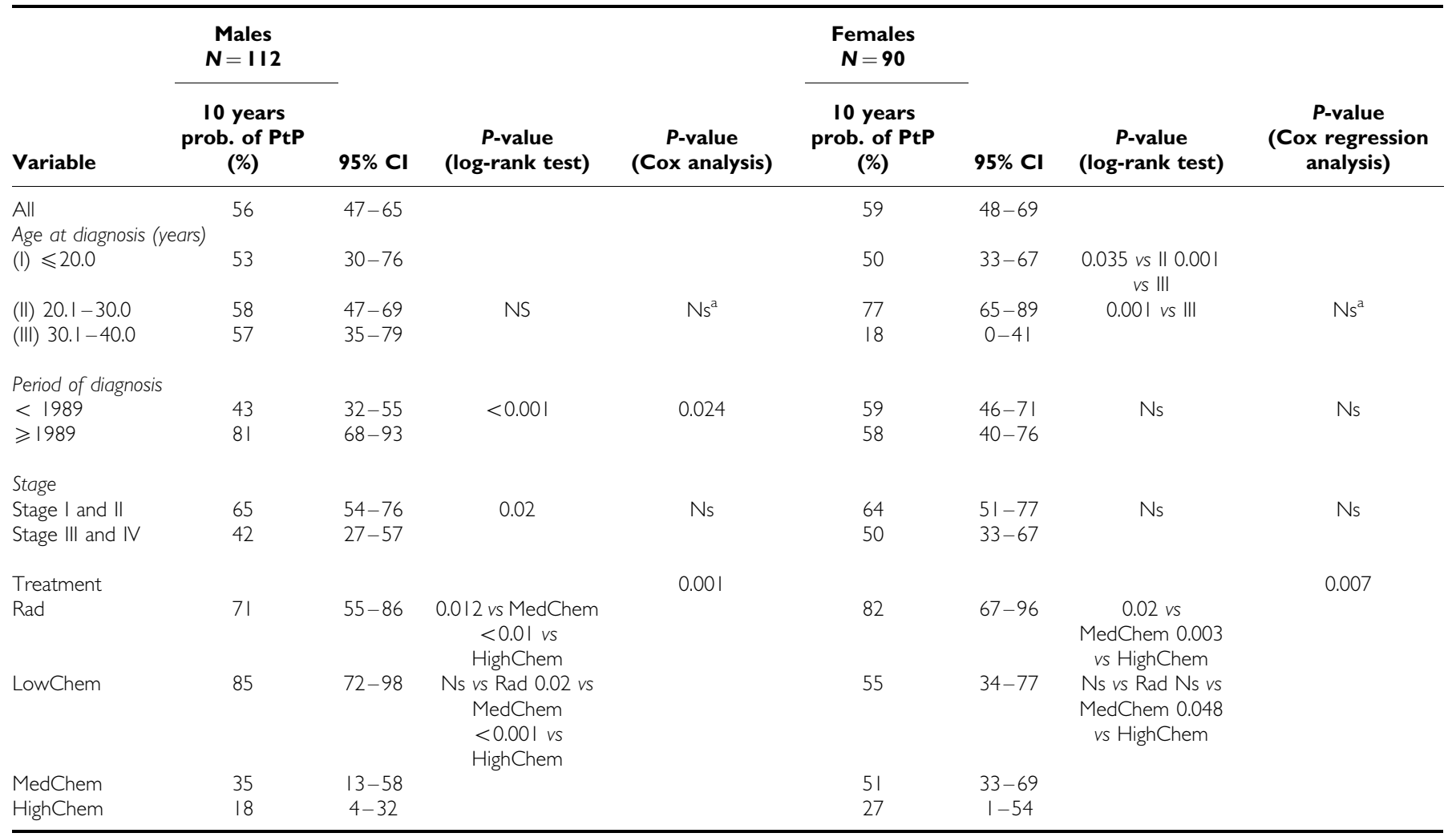

HighChem = high gonadotoxic chemotherapy; lowChem = low gonadotoxic chemotherapy; MedChem = medium gonadotoxic chemotherapy; NS = not significant; PtP = posttreatment parenthood; Rad = Radiotherapy only ${ }^{a}$ Age at diagnosis as continuous variable in Cox regression analysis. Treatment: variable discriminating patients treated with radiotherapy only from those having low, medium or high gonadotoxic chemotherapy.

Table 4 Children conceived by female HLSs treated with high gonadotoxic chemotherapy, for those who had attempted post-treatment parenthood $(N=\mid 1)$

\begin{tabular}{|c|c|c|c|c|c|c|c|}
\hline $\begin{array}{l}\text { Patient } \\
\text { no. }\end{array}$ & $\begin{array}{l}\text { Year of } \\
\text { diagnosis }\end{array}$ & $\begin{array}{l}\text { Age at } \\
\text { diagnosis } \\
\text { (years) }\end{array}$ & Chemotherapy & Radiotherapy (RT) & $\begin{array}{l}\text { Achieved } \\
\text { PtP }\end{array}$ & $\begin{array}{l}\text { Age at birth of first } \\
\text { child post- } \\
\text { treatment (years) }\end{array}$ & $\begin{array}{l}\text { No of children } \\
\text { post-treatment }\end{array}$ \\
\hline 2 & 1979 & 18 & Six MVPP & SupRad & Yes & 29 & Two \\
\hline 3 & 1988 & 17 & Six ChIVPP 2 ABOD & SupRad & Yes & 27 & Two \\
\hline 4 & 1976 & 16 & Six MVPP & $\begin{array}{l}\text { SupRad+Para-aortic } \\
\text { field }\end{array}$ & Yes & $33^{b}$ & Two \\
\hline 6 & 1988 & 19 & Eight ChIVPP & SupRad & Yes & 28 & One \\
\hline 7 & 1988 & 36 & Eight ChIVPP & No RT & No & & \\
\hline 8 & 1990 & 35 & $\mathrm{HDT}^{\mathrm{c}}$ & SupRad & No & & \\
\hline 9 & 1978 & 31 & Six MVPP & SupRad & No & & \\
\hline 10 & 197| & 32 & $\begin{array}{l}\text { Eight MVPP8 CCNU } \\
\text { po }\end{array}$ & $\begin{array}{l}\text { Th10-12 } 3200 \mathrm{rtg} / 10 \\
\text { days }\end{array}$ & No & & \\
\hline II & 1978 & 30 & Six MVPP & $\begin{array}{l}\text { SupRad+Paraaortic } \\
\text { field }\end{array}$ & No & & \\
\hline
\end{tabular}

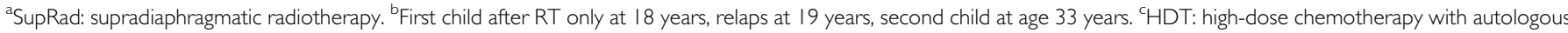
stem cell support.

attempted parenthood after pelvic irradiation, 11 males (four: inverted Y field; four: mantle field/inverted Y field; three: mantle field/L-field) and five females (four: L-field; one: inverted Y field preceded by oophoropexy) were successful.
In the Cox regression multivariate analysis, post-treatment parenthood was significantly associated with treatment group in both males and females. In addition, the period of diagnosis was a significant factor in males. 


\section{Use of ARTs, gamete donation and adoptions}

Thirteen men used ARTs with pretreatment cryopreserved semen, with 10 becoming fathers. Two women used IVF/ICSI, with one of them giving birth to one child.

Thus, at the end of the observation time, 69 (76\%) of the females and $85(71 \%)$ of the males who had attempted post-treatment parenthood had become biological parents after treatment, females having a mean of 1.83 children (range 1-4) and males 1.73 children (range 1-5) post-treatment.

In addition, three males achieved fatherhood with use of donor sperm cells, whereas one female had used oocyte donation (in another Nordic country) without success. Four females and 11 males had adopted a total of 24 children (range 1-3).

\section{DISCUSSION}

Our study is the first to investigate long-term post-treatment parenthood in a large series of consecutive HLSs. About $47 \%$ of the HLSs in this study had attempted post-treatment parenthood. Young age and childlessness at time of diagnosis were significantly associated with attempts to conceive children after treatment. Of those who had attempted post-treatment parenthood, $68 \%$ became parents spontaneously. In addition, 10 males became fathers with use of pretreatment cryopreserved semen and one female conceived with use of ARTs. In multivariate analyses, type of treatment was significantly associated with achievement of posttreatment parenthood in both genders with highest probabilities after radiotherapy only and low gonadotoxic chemotherapy. In addition, an independent and significantly higher success rate was observed in males diagnosed after compared to before 1989.

Several studies have dealt with post-treatment gonadal function in HLSs, most of them with the end point of secondary amenorrhoea, spermatogenesis mirrored by sperm count analyses and the level of serum FSH (Viviani et al, 1985; Viviani et al, 1991; Hill et al, 1995; Behringer et al, 2005). For males, these analyses are a relatively appropriate parameter of fertility, whereas regular post-treatment menstruation does not equal ovulation and the possibility of pregnancy. Some authors have reported the number of childbirths achieved in HLSs, but often in small series (Schilsky et al, 1981; Whitehead et al, 1983; Bonadonna et al, 1984; Anselmo et al, 1990), and rarely with those attempting post-treatment parenthood as a denominator (Aisner et al, 1993; Hodgson et al, 2006).

Compared to these reports, our study has several methodological strengths besides its large size: our end point is the first post-treatment childbirth. Secondly, we relate our end point to the number of patients reported having attempted parenthood. For male HLSs, the proportion of $45 \%$ who reported attempted parenthood is comparable to that observed in two studies of testicular cancer survivors (TCSs), where 31 and 39\%, respectively, had tried to father a child following treatment (Rieker et al, 1990; Brydoy et al, 2005).

However, our number of HLSs who have attempted posttreatment parenthood is probably underestimated for several reasons. Firstly, only surviving patients reported their attempts at parenthood. In studies on future plans for paternity in TCSs, $67 \%$ could not exclude post-treatment fatherhood at the time of diagnosis and $56 \%$ of cancer survivors responded that they would like to have a child in the future, both numbers being higher than in our study (Aass and Fossa, 1988; Schover et al, 1999). The difference may in part be owing to psychological mechanisms in those patients who did not succeed in having children, and expressing that they did not want children may thus be a part of a coping strategy. Furthermore, we have not systematically assessed the reasons why individual patients did not attempt to have children nor how hard the others tried to become a parent. This note of caution is supported by answers from 23 females who stated that they had not attempted post-treatment motherhood because they were convinced of being infertile after cancer treatment. Similar attitudes might be considered in females with premature ovarian failure and in males after intensive treatment.

Reported rates of parenthood after treatment for cancer will depend on selection criteria. In the present study, of those who had attempted post-treatment parenthood, $68 \%$ conceived children spontaneously. As the proportion of patients who reported to have attempted parenthood may be underestimated, our reproduction rates may be too favourable. A study of 43 premenopausal female and 51 male HLSs attempting conception after treatment reported a significantly higher frequency of achieved pregnancies in females than in males, 81 and $49 \%$ respectively. The higher pregnancy rate in females may have been overestimated because HLSs with early menopause may have been excluded from the study (Aisner et al, 1993). The importance of selection bias is emphasised by the figures of a registry-based study from our group, showing a 20 -year probability of first post-treatment childbirth of $8 \%$ in female and $28 \%$ in male lymphoma patients aged $15-45$ years at diagnosis, if all patients treated at the RRMC are included in the denominator (Fossa and Magelssen, 2004). The relatively high grade of success in achievement of post-treatment parenthood in our study may also be explained by the high proportion of patients $(62 \%)$ with initial stage I/II and only $12 \%$ treated for relapse.

In both males and females, achievement of post-treatment parenthood was associated with treatment, with the type and intensity of chemotherapy being most important. This corresponds to earlier studies on male fertility after treatment for HL, with higher rates of preserved fertility after treatment with ABVDlike regimens compared to MOPP-like regimens (Viviani et al, 1985; Anselmo et al, 1990; Viviani et al, 1991; Hill et al, 1995). Two male patients spontaneously fathered children after HDT. A recent report found post-treatment conceptions in $7 \%$ of males after HDT for HL and NHL, but neither reported the use of ARTs nor attempts of post-treatment parenthood (Carter et al, 2006). Only a small proportion of the HLSs in this study used their pretreatment cryopreserved semen, which has also been shown for other cancer survivors (Ragni et al, 2003). However, 10 of 13 males successfully achieved fatherhood with their cryopreserved semen, which is more promising than in TCSs (Magelssen et al, 2005).

Earlier reports have shown that female post-treatment fertility depends on the degree of gonadotoxicity of the treatment given and age at diagnosis (Schilsky et al, 1981; Whitehead et al, 1983; Bonadonna et al, 1984; Behringer et al, 2005). In a recent publication, the 12 -month pregnancy rate was $70 \%$ in 36 female HLSs attempting pregnancy after treatment with ABVD, with no significant difference compared to the control group $(n=29)$ (Hodgson et al, 2006). In our study, age at diagnosis in females was a significant factor associated with post-treatment parenthood in univariate analysis. Age determines the remaining oocyte reserve (Faddy et al, 1992), so the probability of sufficient oocyte reserve decreases with increasing age.

No differences were found in post-treatment parenthood after supradiaphragmatic radiotherapy only compared to infradiaphragmatic radiotherapy with or without supradiaphragmatic irradiation. However, almost $80 \%$ of the irradiated patients had received supradiaphragmatic radiotherapy only, and the group of HLSs who had attempted post-treatment parenthood after infradiaphragmatic radiotherapy was too small for valid statistical comparison. As shown previously, successful pregnancies are possible even after pelvic irradiation if oophoropexy has been performed before inverted Y field irradiation (Le et al, 1976) or the radiation field did not include both ovaries.

The improved probability of post-treatment parenthood in males diagnosed after 1989 compared to before 1989 is as expected, owing to changes in the treatment of HL in late 1980s with introduction of fertility-saving treatment as less gonadotoxic 
chemotherapy and less extensive radiation fields. Changes in attitudes towards life after treatment for HL and a more optimistic view among health professionals with regard to having children after cancer treatment in the latter period may also have contributed to the increased paternity rate.

Our study has some limitations: we do not know at what time after treatment the HLSs started attempting parenthood, how seriously they attempted this goal or the fertility status of our patient's partners. Finally, we assume that each male HLS who reported spontaneous post-treatment fatherhood is the biological father of the child. Exclusion of patients with relapse after 1999 or a second cancer from the survey and the lack of relevant information in deceased patients may have led to loss of information about patients trying to have children in the years between initial treatment and relapse, second cancer or death.

Information on fertility issues is important in clinical oncological practice, and fertility-saving tasks should be discussed with patients at risk of post-treatment infertility. Females should be informed that both the treatment and their age at treatment influence their fertility potential. Females aged above 30 years at diagnosis are at high risk of becoming infertile. They constitute a subgroup for whom cryopreservation of ovarian tissue should be considered. As males have the opportunity for pretreatment cryopreservation of semen and as spermatogenesis recovers in most of them, their potential infertility after treatment is easier to deal with.

\section{ACKNOWLEDGEMENTS}

We thank Trine Bjøro, Central Laboratory, RikshospitaletRadiumhospitalet Medical Center, Oslo, Norway. This project has been financed with the aid of EXTRA funds from the Norwegian Foundation for Health and Rehabilitation.

\section{REFERENCES}

Aass N, Fossa SD (1988) Paternity in young patients with testicular cancer expectations and experience. Prog Clin Biol Res 269: 481-491

Abrahamsen AF, Egeland T, Hansen S, Langholm R, Holte H, Kvaloy S (1997) Hodgkin's disease in a national and hospital population: trends over 20 years. Eur J Cancer 33: $2380-2383$

Abrahamsen AF, Hannisdal E, Nome O, Holte H, Hager B, Langholm R, Kvaloy S (1996) Clinical stage I and II Hodgkin's disease: long-term results of therapy without laparotomy. Experience at one institution. Ann Oncol 7: 145-150

Aisner J, Wiernik PH, Pearl P (1993) Pregnancy outcome in patients treated for Hodgkin's disease. J Clin Oncol 11: 507-512

American Society of Clinical Oncology, Lee SJ, Schover LR, Partridge AH, Patrizio P, Wallace WH, Hagerty K, Beck LN, Brennan LV, Oktay K (2006) American Society of Clinical Oncology recommendations on fertility preservation in cancer patients. J Clin Oncol 24: 2917-2931

Anselmo AP, Cartoni C, Bellantuono P, Maurizi-Enrici R, Aboulkair N, Ermini M (1990) Risk of infertility in patients with Hodgkin's disease treated with ABVD vs MOPP vs ABVD/MOPP. Haematologica 75: $155-158$

Aurlien E, Holte H, Pharo A, Kvaloy S, Jakobsen E, Smeland EB, Kvalheim G (1998) Combination chemotherapy with mitoguazon, ifosfamide, MTX, etoposide (MIME) and G-CSF can efficiently mobilize PBPC in patients with Hodgkin's and non-Hodgkin's lymphoma. Bone Marrow Transplant 21: $873-878$

Behringer K, Breuer K, Reineke T, May M, Nogova L, Klimm B, Schmitz T, Wildt L, Diehl V, Engert A, German Hodgkin's Lymphoma Study Group (2005) Secondary amenorrhea after Hodgkin's lymphoma is influenced by age at treatment, stage of disease, chemotherapy regimen, and the use of oral contraceptives during therapy: a report from the German Hodgkin's Lymphoma Study Group. J Clin Oncol 23: $7555-7564$

Blystad AK, Holte H, Kvaloy S, Smeland E, Delabie J, Kvalheim G (2001a) High-dose therapy in patients with Hodgkin's disease: the use of selected $\mathrm{CD} 34(+)$ cells is as safe as unmanipulated peripheral blood progenitor cells. Bone Marrow Transplant 28: 849-857

Blystad AK, Torlakovic E, Holte H, Kvaloy S, Lenschow E, Kvalheim G (2001b) CD34(+) cell enrichment depletes atypical CD30(+) cells from PBPC grafts in patients with HD. Cytotherapy 3: 295-305

Bonadonna G, Santoro A, Viviani S, Lombardi C, Ragni G (1984) Gonadal damage in Hodgkin's disease from cancer chemotherapeutic regimens. Arch Toxicol 7: $140-145$

Brydoy M, Fossa SD, Klepp O, Bremnes RM, Wist EA, Wentzel-Larsen T, Dahl O (2005) Paternity following treatment for testicular cancer. J Natl Cancer Inst 97: 1580-1588

Carter A, Robison LL, Francisco L, Smith D, Grant M, Baker KS, Gurney JG, McGlave PB, Weisdorf DJ, Forman SJ, Bhatia S (2006) Prevalence of conception and pregnancy outcomes after hematopoietic cell transplantation: report from the Bone Marrow Transplant Survivor Study. Bone Marrow Transplant 37: 1023-1029
Diehl V, Mauch P, Harris NL Chapter 45.6., 2339-2387 Hodgkin's disease. Cancer 2001 6th Edition Philadelphia: Lippincott

Faddy MJ, Gosden RG, Gougeon A, Richardson SJ, Nelson JF (1992) Accelerated disappearance of ovarian follicles in mid-life: implications for forecasting menopause. Hum Reprod 7: 1342-1346

Fossa SD, Magelssen H (2004) Fertility and reproduction after chemotherapy of adult cancer patients: malignant lymphoma and testicular cancer. Ann Oncol 15(Suppl): 65

Hill M, Milan S, Cunningham D, Mansi J, Smith I, Catovsky D, Gore M, Zulian G, Selby P, Horwich A (1995) Evaluation of the efficacy of the VEEP regimen in adult Hodgkin's disease with assessment of gonadal and cardiac toxicity. J Clin Oncol 13: 387-395

Hjermstad MJ, Fossa SD, Oldervoll L, Holte H, Jacobsen AB, Loge JH (2005) Fatigue in long-term Hodgkin's disease survivors: a follow-up study. J Clin Oncol 23: 6587-6595

Hjermstad MJ, Oldervoll L, Fossa SD, Holte H, Jacobsen AB, Loge JH (2006) Quality of life in long-term Hodgkin's disease survivors with chronic fatigue. Eur J Cancer 42: $327-333$

Hodgson DC, Pintilie M, Gitterman L, Dewitt B, Buckley CA, Ahmed S, Smith K, Schwartz A, Tsang RW, Crump M, Wells W, Sun A, Gospodarowicz MK (2006) Fertility among female hodgkin lymphoma survivors attempting pregnancy following ABVD chemotherapy. Hematol Oncol 25: $11-15$

Holte H, Mella O, Wist E, Telhaug R, Hannisdal E, Abrahamsen AF (1996) ChlVPP is as effective as alternating ChlVPP/ABOD in advanced stage Hodgkin's disease. Acta Oncologica 35(Suppl): 80

Howell SJ, Shalet SM (2001) Testicular function following chemotherapy. Hum Reprod Update 7: 363 - 369

Le FO, Donaldson SS, Kaplan HS (1976) Pregnancy following oophoropexy and total nodal irradiation in women with Hodgkin's disease. Cancer 38: $2263-2268$

Magelssen H, Haugen TB, von DV, Melve KK, Sandstad B, Fossa SD (2005) Twenty years experience with semen cryopreservation in testicular cancer patients: who needs it? Eur Urol 48: 779-785

Mills W, Chopra R, McMillan A, Pearce R, Linch DC, Goldstone AH (1995) BEAM chemotherapy and autologous bone marrow transplantation for patients with relapsed or refractory non-Hodgkin's lymphoma. J Clin Oncol 13: $588-595$

Ragni G, Somigliana E, Restelli L, Salvi R, Arnoldi M, Paffoni A (2003) Sperm banking and rate of assisted reproduction treatment: insights from a 15-year cryopreservation program for male cancer patients. Cancer 97: 1624-1629

Rieker PP, Fitzgerald EM, Kalish LA (1990) Adaptive behavioral responses to potential infertility among survivors of testis cancer. J Clin Oncol 8: $347-355$

Schilsky RL, Sherins RJ, Hubbard SM, Wesley MN, Young RC, DeVita VT (1981) Long-term follow up of ovarian function in women treated with MOPP chemotherapy for Hodgkin's disease. Am J Med 71: $552-556$ 
Schover LR, Rybicki LA, Martin BA, Bringelsen KA (1999) Having children after cancer. A pilot survey of survivors' attitudes and experiences. Cancer 86: 697 - 709

Viviani S, Ragni G, Santoro A, Perotti L, Caccamo E, Negretti E, Valagussa P, Bonadonna G (1991) Testicular dysfunction in Hodgkin's disease before and after treatment. Eur J Cancer 27: 1389-1392
Viviani S, Santoro A, Ragni G, Bonfante V, Bestetti O, Bonadonna G (1985) Gonadal toxicity after combination chemotherapy for Hodgkin's disease. Comparative results of MOPP vs ABVD. Eur J Cancer Clin Oncol 21: 601 - 605

Whitehead E, Shalet SM, Blackledge G, Todd I, Crowther D, Beardwell CG (1983) The effect of combination chemotherapy on ovarian function in women treated for Hodgkin's disease. Cancer 52: 988-993 